\title{
Variation in the fatty acid profiles of two cold water diatoms grown under different temperature, light, and nutrient regimes
}

\author{
Kristian Spilling ${ }^{1,2}$ (D) Jukka Seppälä ${ }^{1} \cdot$ Dagmar Schwenk $^{3} \cdot$ Heiko Rischer $^{3} \cdot$ Timo Tamminen $^{1}$
}

Received: 27 October 2020 / Revised and accepted: 3 January 2021 / Published online: 16 February 2021

(C) The Author(s) 2021

\begin{abstract}
There is a growing demand for marine omega- 3 fatty acids (FAs) that is produced in high amounts by some microalgae. Here we determined the FA profiles of two cold water adapted diatoms, Chaetoceros wighamii and Thalassiosira baltica. The cultures were acclimated to different temperatures $\left(3,7,11,15\right.$, and $\left.19^{\circ} \mathrm{C}\right)$ and irradiance $\left(20,40,130\right.$, and $450 \mu \mathrm{mol}$ photons $\left.\mathrm{m}^{-2} \mathrm{~s}^{-1}\right)$ and the FA profiles were determined in exponential and stationary growth phases, the latter induced by different nutrient limitation (N, P, and Si). The maximum growth rate was obtained by both species at $11^{\circ} \mathrm{C}, \geq 130 \mu \mathrm{mol}$ photons $\mathrm{m}^{-2} \mathrm{~s}^{-1}$ and was 0.8 day $^{-1}$ and 0.6 day $^{-1}$ for $C$. wighamii and T. baltica, respectively. Both species contained relatively high amounts of eicosapentaenoic acid (EPA). Thalassiosira baltica accumulated maximally $\sim 30 \mathrm{mg} \mathrm{EPA} \mathrm{g}^{-1}$ ash-free dry weight (AFDW) under Si-limitation. The content of docosahexaenoic acid (DHA) was lower, reaching up to $4 \mathrm{mg}^{\mathrm{DHA} \mathrm{g}} \mathrm{g}^{-1} \mathrm{AFDW}$ in $T$. baltica. The concentration of EPA correlated positively with the chlorophyll $a$ :carbon ratio, suggesting that it is bound to membranes in the photosynthetic apparatus and the EPA content in T. baltica was high enough to consider it as a potent candidate for cultivation under cold $\left(<15^{\circ} \mathrm{C}\right)$ conditions. Covering a wide range of environmental conditions, the strongest differentiation in FA profiles was observed between the species with the growth phase/nutrient limitation pattern as the second most important driver of the FA composition.
\end{abstract}

Keywords Bacillariophyceae $\cdot$ Microalgae $\cdot$ Lipids $\cdot$ Omega-3 $\cdot 20: 5 \mathrm{n} 3 \cdot 22: 6 \mathrm{n} 3 \cdot$ Baltic sea

\section{Introduction}

Microalgae constitute an important source of fatty acids (FAs) in nature, and, with their high growth rate under optimal conditions, the cultivation of microalgae for commercial use has gained increasing interest over the past decades. There has been a lot of focus on developing biofuels from algal lipids (e.g., Williams and Laurens 2010), but also much higher value products can be extracted from microalgae. Omega-3 FAs have for example a significant market as dietary supplements valued at $>2$ billion US\$ (GVR 2020). Microalgae can produce relatively large amounts of omega-3 FAs, but the concentration depends on both

Kristian Spilling

kristian.spilling@environment.fi

1 Marine Research Centre, Finnish Environment Institute, Agnes Sjöbergin katu 2, 00790 Helsinki, Finland

2 Department of Natural Sciences, University of Agder, Kristiansand, Norway

3 VTT Technical Research Centre of Finland Ltd., Tietotie 2, 02044 Espoo, Finland the species and environmental conditions during growth (Guschina and Harwood 2009). There is a range of literature on different species and how growth limitations can increase the total FA content and specific FAs (e.g., Guschina and Harwood 2009; Jónasdóttir 2019).

Lipids are a diverse group of biomolecules built of FAs and have a range of intracellular functions in phytoplankton such as structural elements of cell membranes, as, for example, phospholipids and galactolipids, and as carbon storage forms such as in triacylglycerol (TAGs). The FAs are in turn made of hydrocarbon chains and can be further divided into saturated (SFA), monounsaturated (MUFA), and polyunsaturated (PUFA) FAs depending on whether there are none, one, or two or more double bonds in the carbon chain, respectively. For a review of the lipid synthesis in algae, see, e.g., Khozin-Goldberg (2016).

The FA eicosapentaenoic acid (EPA) and docosahexaenoic acid (DHA) are the most conspicuous examples of marine PUFAs that are generally not produced by terrestrial plants, and both EPA and DHA have beneficial properties as part of a human diet (Swanson et al. 2012). Light and temperature affect the FA profile as, e.g., photosynthetic pigments, and the membranes surrounding them increase at low light 
(Falkowski and Raven 1997). In diatoms, these membranes seem to consist to a large extent of PUFAs, in particular EPA (Xu et al. 2010), in contrast to higher plants that to a larger extent have shorter chain PUFAs (18:3 and 16:3) in their plastid membranes (Douce and Joyard 1996). The light-dark cycle might also affect the FA profile, with continuous light providing more TAGs (Brown et al. 1996).

The FA synthesis in diatoms is rapidly up- or downregulated as a response to shifts in temperature and it relates to the regulation of the cellular metabolism (Liang et al. 2019). In addition, changes in temperature may affect the fluidity of the lipids bound to cellular membranes, and one of the ways to cope with decreasing temperatures is to increase the proportion of PUFAs that are more fluid than SFAs (Jiang and Gao 2004; Svenning et al. 2019). Although an increasing proportion of PUFAs with decreasing temperature seems to be a general phenomenon, there are large species-specific differences and this correlation does not necessarily exist in all species (Thompson et al. 1992).

During exponential growth algae channel their resources into growth, but when growth stops due to inorganic nutrient limitation, photosynthesis may still fix carbon that some species store in the form of lipids. Nitrogen limitation, in particular, seems to enhance storage lipid accumulation in TAGs and most of the FAs synthesized are generally SFAs (e.g., 16:0) and MUFAs (e.g., 16:1) (Guschina and Harwood 2009).

Most of the work on algal FAs has been performed at temperatures $>10^{\circ} \mathrm{C}$ and there is at present a gap in profiling FAs of cold water adapted phytoplankton (Artamonova et al. 2017). Considering algal cultivation in temperate areas, cultivating cold water species could be beneficial during wintertime when the air temperature decreases $<10{ }^{\circ} \mathrm{C}$. When the temperature decreases close to freezing, cold water adapted phytoplankton can still obtain high growth rates (Schwenk et al. 2013). Understanding how temperature affects the FA profiles would be important for species selection and optimizing the cultivation system.

Based on our previous work with cold water diatoms (Schwenk et al. 2013), we focus here on two species exhibiting high growth rates at low temperatures. Our main aim was to examine the variability in the FA profiles depending on temperature and light and comparing exponential growth with different inorganic nutrient (N, P, and Si) limitation regimes. We were particularly interested in the content of the long-chained PUFAs: EPA and DHA.

\section{Materials and methods}

\section{Culture acclimation and growth}

Chaetoceros wighamii and Thalassiosira baltica were sourced from the culture collection of the Tvärminne
Zoological Station (strains TVCWI, TVTBA) and cultured in T2 medium at 6 PSU, which is a modified F/2 medium (Guillard 1975) with N:Si:P nutrient ratios adjusted to $16: 8: 1$, previously suggested to be optimal for these diatoms (Spilling 2007). The cultures were grown in 2-L polycarbonate flasks (filled to $1.5 \mathrm{~L}$ ) and acclimated to different temperature $\left(3,7,11,15,19\right.$, and $\left.23^{\circ} \mathrm{C}\right)$ and irradiance $(20,40,130$, and $450 \mu \mathrm{mol}$ photons $\mathrm{m}^{-2} \mathrm{~s}^{-1}$ from daylight fluorescent tubes, Philips TLD 965). The flasks were incubated in a temperature-regulated water bath and irradiance was adjusted with neutral density screens. During the temperature acclimation phase, we started at the lowest temperature, and during exponential growth, a subsample was inoculated into the following temperature. This was done in $4{ }^{\circ} \mathrm{C}$ increments until no growth was detected. The temperature range was different for the two species and the maximum temperature with growth was $11^{\circ} \mathrm{C}$ and $19^{\circ} \mathrm{C}$ for C. wighamii and T. baltica, respectively. The cultures were kept in suspension by bubbling with pre-filtered $(0.2 \mu \mathrm{m})$ air. Cell growth was monitored daily by counting cells with a FlowCam (FluidImaging), which collects micrographs of individual cells passing through a flow cuvette.

On sampling days, a subsample was filtered for determining the dry weight (DW), ash-free dry weight (AFDW), particulate organic carbon (POC), and chlorophyll $a$ (Chla). In addition, an aliquot was centrifuged $(10,000 \mathrm{rpm})$, the pellet frozen, and stored at $-80^{\circ} \mathrm{C}$ prior to the determination of FAs.

For DW and AFDW, pre-rinsed and combusted 25-mm GF/F filters (Whatman) were used. The filters had been individually weighted before filtration and kept in plastic Petri dishes. A known volume (25-200 mL, depending on the biomass concentration) was filtered onto the filters and they were subsequently dried in an oven $\left(60^{\circ} \mathrm{C}\right)$ overnight $(>8 \mathrm{~h})$. The filters were weighed again to determine the DW. The filters were combusted at $450^{\circ} \mathrm{C}(4 \mathrm{~h})$ and the ash weight (AW) was determined by weighing the filters again. Finally, the AFDW was determined by subtracting the AW from the DW. For determining the POC, subsamples were filtered onto acidwashed and pre-combusted GF/F filters. These were dried and stored at room temperature before measurement of the POC with a mass spectrometer according to Koistinen et al. (2017). For Chla, we used GF/F filters and the Chla was extracted in $96 \%$ ethanol for $24 \mathrm{~h}$ at room temperature following the protocol of Jespersen and Christoffersen (1987). The Chla content was determined with a spectrofluorometer (Cary Varian Eclipse) calibrated against pure Chla standards (Sigma) using 430$\mathrm{nm}$ excitation and recording the 670-nm emission light.

The exponential growth sampling was carried out when the biomass was $10-50 \%$ of the maximum (in terms of POC). After the sampling, all but $100 \mathrm{~mL}$ of the culture was removed and a new medium with limiting N, P, or Si was added. This procedure was done for a subsample of initial treatments, 
representing five combinations of temperature and light conditions (Supplementary Table S1). All but the limiting nutrient (kept at the original concentration) were added in 5-fold concentrations. Growth was monitored as described above until biomass did not increase over three consecutive days. The second set of measurements was taken during this early stationary growth phase.

\section{Determination of fatty acids}

We added $500 \mu \mathrm{L}$ of chilled $\left(-20^{\circ} \mathrm{C}\right)$ methanol with $0.1 \%$ butylated hydroxytoluene to the 5-mg DW algae sample in order to break the cells. The resulting solution was incubated at $-20^{\circ} \mathrm{C}$ for $10 \mathrm{~min}$ in reaction vials. The algal samples were further disrupted with two 4-mm stainless steel balls in a mixer mill (Retsch MM 301) for 3 min at $25 \mathrm{~Hz}$. Temperature for these first steps was kept $<0^{\circ} \mathrm{C}$ by pre-chilling all equipment.

The following steps were performed at room temperature: $1000 \mu \mathrm{L}$ chloroform and $150 \mu \mathrm{L}$ internal standard $(1549 \mathrm{mg}$ $\mathrm{L}^{-1}$ triheptadecanoin, Sigma-Aldrich, $1029.6 \mathrm{mg} \mathrm{L}^{-1}$ heptadecanoic acid in chloroform:methanol, 2:1, Fluka, Sigma-Aldrich) was added to each sample and mixed for 10 min. After centrifugation, the supernatant was acidified with $300 \mu \mathrm{L}, 20 \mathrm{mM}$ acetic acid, mixed for 5 to $10 \mathrm{~min}$, and centrifuged again. The organic phase was extracted twice using $500 \mu \mathrm{L}$ chloroform, mixing for $10 \mathrm{~min}$, and centrifugation. Organic phases were pooled and a subsample of $750 \mu \mathrm{L}$ was dried in a glass tube under nitrogen flow. The residue was dissolved in $300 \mu \mathrm{L}$ isopropanol. Each sample was extracted and the FAs were determined in duplicates. The average of these measurements was used for the data analysis.

The FAs were determined using transmethylation and gas chromatography (GC) with a flame ionization detector (FID). The samples were dried again under nitrogen flow, taken up in $700 \mu \mathrm{L}$ petroleum-ether, and $125 \mu \mathrm{M}$ sodium methoxide (97\%, dissolved in methanol) was added before boiling the solution at $45^{\circ} \mathrm{C}$ for $5 \mathrm{~min}$. After cooling, $500 \mu \mathrm{L} \mathrm{NaHSO}_{4}(15 \% \mathrm{~m} / \mathrm{v})$ and $200 \mu \mathrm{L}$ petroleum-ether were added and the samples were mixed. After separation of the two phases by centrifugation, the petroleum-ether phase was transferred to a $\mathrm{GC}$ vial. The solvent was evaporated and the residue was dissolved in $1000 \mu \mathrm{L}$ hexane. Subsequently, $1 \mu \mathrm{L}$ of this solution was used for further gas chromatography (GC) analysis. This transmethylation procedure does not methylate the free fatty acids (FFA), allowing distinction of FFA and structural FAs in the following GC step.

FAs were separated and evaluated quantitatively by using a capillary GC (7890A with sampler CTC ANALYTICS GCPAL SYSTEM, Agilent Technologies) equipped with a BP21 column $(25 \mathrm{~m} \times 0.2 \mathrm{~mm} \times 0.3 \mathrm{~mm}$, HP-FFAP Polyethylene Glycol TP, Agilent) and connected to a FID. The injector temperature was $260^{\circ} \mathrm{C}$. For every sample, $1 \mu \mathrm{L}$ was injected and transferred splitless to the column. The oven temperature was programmed to increase from $70(1.5 \mathrm{~min})$ to $240{ }^{\circ} \mathrm{C}$ at the rate of $7{ }^{\circ} \mathrm{C} \mathrm{min}^{-1}$. The carrier gas was helium with pressure of 16.671 psi. Peaks were allocated to substances via multicapillary column gas chromatography and via comparison to reference substances (F.A.M.E. Mix, \#1891, Supelco, Sigma-Aldrich). Altogether, 30 FAs or FA methyl esters were identified and quantified.

\section{Data treatment}

Statistical analysis was carried out using SigmaPlot 13. Student's $t$ test was used to test for differences between two groups. For comparing more groups, we used analysis of variance (ANOVA) followed by a post hoc test: Tukey, when the variance between groups was similar, or on ranks followed by Dunn's method if the variances between groups were different.

An ordination plot (non-metric multidimensional scaling, NMDS) was used to identify links between the species, growth conditions, and FA profile. The coordinates of each sampling along the NMDS axes 1 and 2 were used as explanatory variables in separate generalized additive models (GAMs), using R packages "vegan" (metaMDS function) and "mgcv" (Wood 2016). The response variables were used to color the symbols in the resulting NMDS plots, using R packages "colorRamps" (Keitt 2008) and "plotrix" (Lemon 2006).

\section{Results}

For both species, the maximum growth rate was obtained at 11 ${ }^{\circ} \mathrm{C}$ and an irradiance of $\geq 130 \mu \mathrm{mol}$ photons $\mathrm{m}^{-2} \mathrm{~s}^{-1}$, but the species differed in growth rate with increasing temperature (Fig. 1). Chaetoceros wighamii had the highest maximum growth rate at $\sim 0.8 \mathrm{day}^{-1}$, and $11^{\circ} \mathrm{C}$ was the maximum temperature where it grew. Thalassiosira baltica had a slightly lower maximum growth rate at $\sim 0.6$ day $^{-1}$ and grew at lower rates up to $19^{\circ} \mathrm{C}$.

During exponential growth, the harvested biomass was similar for both species at roughly $50 \mathrm{mg} \mathrm{AFDW} \mathrm{L}^{-1}$ (Fig. 2). The biomass concentration increased in the stationary growth phase and with the growth medium used, which was adjusted to prevent too high biomass and self-shading (light) effects. The highest biomass harvest was in the N- and Plimited cultures with roughly $250-350 \mathrm{mg}$ AFDW L ${ }^{-1}$. The harvested AFDW was lower during Si-limitation, in particular for $T$. baltica ( $100 \mathrm{mg}$ AFDW L ${ }^{-1}$; Fig. 2$)$.

The share of FAs of the biomass was affected by the growth phase, and there was a clear difference between the species (Fig. 2). Thalassiosira baltica had a higher FA content during exponential growth at $7 \%$ of AFDW compared with $C$. wighamii at $3 \%$ of AFDW ( $t$ test, $p=0.006$ ). For both species, the FA content increased in the stationary growth 


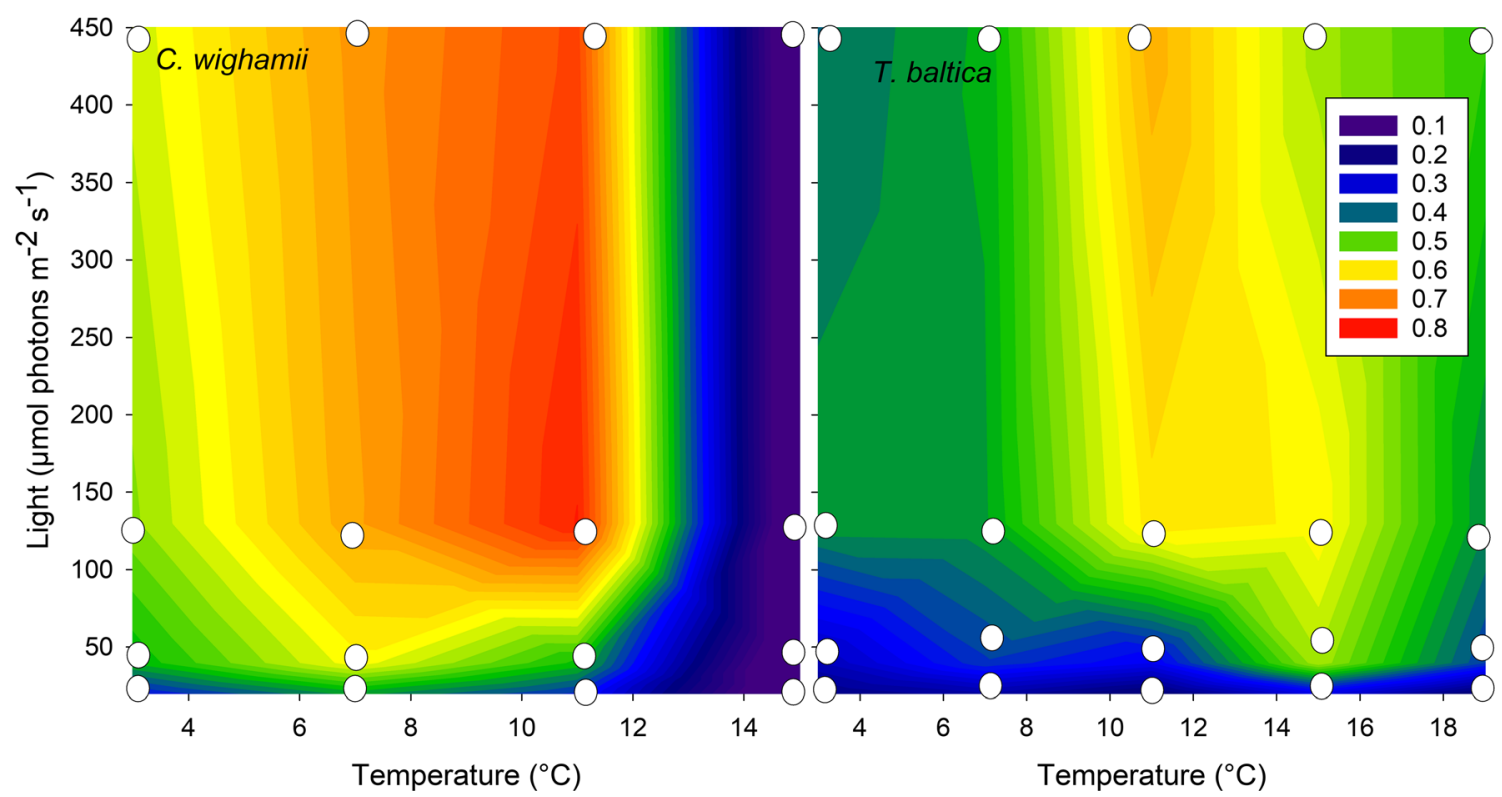

Fig. 1 The growth rate $\left(\mathrm{day}^{-1}\right)$ of Chaetoceros wighamii and Thalassiosira baltica grown under nutrient replete conditions in a different light (16:8 light-dark cycle) and temperature. The white dots

represent the sampling points. $C$. wighamii and T. baltica did not grow at 15 and $23{ }^{\circ} \mathrm{C}$, respectively. The growth rate of $C$. wighamii was redrawn from Spilling et al. (2015)

phase, but for T. baltica, Si-limitation produced the highest average FA content, which was higher than exponential growth and N-limitation (Dunn, $p<0.04$ ) but was statistically not different from P-limitation (Dunn, $p=0.3$ ). For C. wighamii, $\mathrm{N}$-limitation increased the $\mathrm{FA}$ content most (Tukey, $p<0.001$ ) to an average of $46 \%$ of AFDW (Fig. 2).

In ordination, the strongest differentiation in FA profiles was observed between the species $\left(R^{2}=0.77\right.$ of MDS1, $p<$ 0.0001 ; Fig. 3), with the growth phase/limitation pattern as the second most important driver of the sample FA composition (Supplementary Fig S1). The main difference between the species was the much higher proportion of free FAs in C. wighamii while T. baltica contained more of the FAs: 18:4, 20:3, and 22:5 (Fig. 3, table S1).

Although the species and growth phase were the dominating factors influencing the full FA profile, there were also effects of light and temperature in individual FAs. During exponential growth, the share of PUFAs was clearly highest in T. baltica (Fig S2). For this species, there was both a temperature and light effect with a higher proportion of PUFA at low temperature and low light at close to $50 \%$ of the total FAs. In C. wighamii, the overall PUFA content was $<30 \%$ of total FAs during exponential growth and it was highest at low light with no apparent temperature effect (Fig S2, Table S1).

The share of SFA was higher in exponential growth compared with $\mathrm{N}$-limitation (Fig S3, Tukey, $p=0.003$ ) in $C$. wighamii and with Si-limitation in T. baltica (Dunn, $p<0.001$ ) but without statistically significant difference between the other treatments $(p$ $>0.06$ ). Light affected the share of 16:0 and 16:1 FAs with an increasing share of total FAs with increasing irradiance (Fig S4).
The share of MUFA was higher in the stationary growth phase compared with nutrient replete growth for both species $(p \leq 0.02)$ apart from exponential growth and $\mathrm{N}$-limitation in $T$. baltica (Fig S2, Dunn, $p=0.2$ ). Most of this increased share in MUFA was the FA 16:1 (table S1). The share of PUFA was highest in the exponential growth in $T$. baltica compared with all nutrientlimited stationary growth phases (Fig S2, Tukey, $p<0.02$ ). In $C$. wighamii, only a difference between exponential and $\mathrm{N}$ - and P-limited growth in PUFAs was observed (Dunn, $p \leq 0.01$ ).

The highest eicosapentaenoic acid (EPA) content was found in T. baltica (Fig. 4). Out of the total FAs, the highest share of EPA was obtained during exponential growth, but the highest content of EPA in the AFDW was found during Si-limitation and the highest overall concentration during N-limitation (Fig. 4). For C. wighamii, the highest content of EPA was found during Nlimitation both in relation to AFDW biomass and concentration per volume (Fig. 4). The concentration of docosahexaenoic acid (DHA) was lower in both species being $<20 \%$ of the EPA concentration under most conditions, except for $C$. wighamii during exponential growth where it was a bit higher (up to $36 \%$ of the EPA content). The DHA content was highest in T. baltica with up to 4 mg DHA g ${ }^{-1}$ AFDW.

There was a clear positive correlation between the EPA share of the total FAs and the Chla:C ratio (Fig. 5). This was most pronounced for $T$. baltica that had a steeper slope and higher coefficient of determination $\left(R^{2}\right)$ compared with $C$. wighamii. It was also more pronounced in the exponential growth phase as the Chla:C ratio was in the low end during the stationary growth phase with a few exceptions during $\mathrm{Si}$ limitation in C. wighamii. 
Fig. 2 Biomass as ash-free dry weight (AFDW) and total fatty acids at the time of harvesting of Chaetoceros wighamii and Thalassiosira baltica grown under exponential (Exp) and in stationary growth phase under nitrogen- (N-lim), phosphorus(P-lim), and silicate- (Si-lim) limitation. The box is the $25-75 \%$ percentile; the mid-line is the median; the whiskers indicate the $10-90 \%$ percentile and the dots outliers

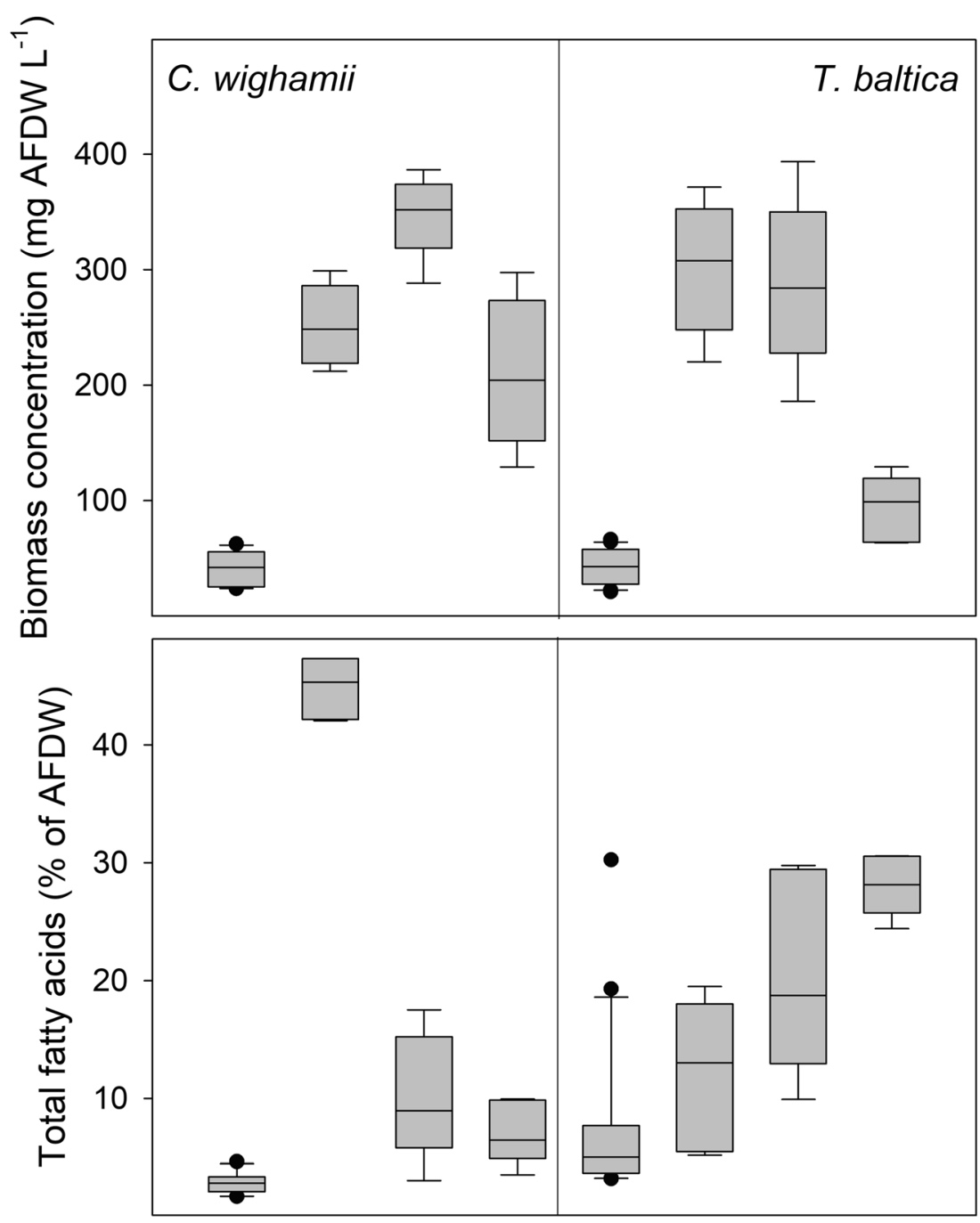

Exp N-lim P-lim Si-lim Exp N-lim P-lim Si-lim

\section{Discussion}

The two diatoms cultivated here are naturally occurring in the Baltic Sea during winter and spring and represent two of the most dominating diatoms during this time period. T. baltica is larger in size than $C$. wighamii but occupies a similar niche in the ecosystem (Spilling 2007). There are some distinctions; however, $T$. baltica has a lower maximum growth rate as revealed in this study and apparently also allocates its carbon differently, e.g., provides less exudates for heterotrophic bacteria in a natural environment (Camarena-Gómez et al. 2021). It is interesting that the FA profiles differ so much implying relevant differences in food quality for higher trophic levels, i.e., a different potential for feed cultivation of these species. From a perspective of extracting omega-3 FAs, T. baltica is the preferred candidate with a very high proportion of EPA. Chaetoceros wighamii would be the better species if SFA is the preferred product, which could be the case for, e.g., paraffinic biodiesel made from hydrotreated vegetable oils.
In the literature there are many ways to present FA data. Most often, individual FAs are presented as a percentage of total FAs or alternatively as a share of a biomass measure such as DW, AFDW, or carbon content. For the latter, some form of DW is often reported and to compare with other studies we related the EPA content primarily to the AFDW. Diatoms exhibit a higher ash content than other microalgal groups due to their silica frustule (Nalewajko 1966), and for this reason, we used AFDW rather than DW. Using DW creates a bias when comparing diatoms with other algal groups and when comparing different diatoms because some species are more silicified than others. Even within the same species, the degree of silicification may differ, e.g., due to Si-limitation, which may shift the FA content per DW unit when the ash content decreases (Steinrücken et al. 2018).

EPA clearly constituted the most prominent PUFA in both the species, and its concentration as a percentage of total FAs was clearly higher at low light acclimation. The positive correlation with the Chla: $\mathrm{C}$ ratio indicates that it 
Fig. 3 Data ordination (NMDS) of the fatty acids (FAs) in the two species Chaetoceros wighamii (red) and Thalassiosira baltica (black), with each of the individual FAs placed into the ordination space. Plots with differentiation between the cultivation variables (light, temperature, and nutrient limitation) can be found in Supplementary Fig S1 and the statistical analysis in Supplementary Table S2

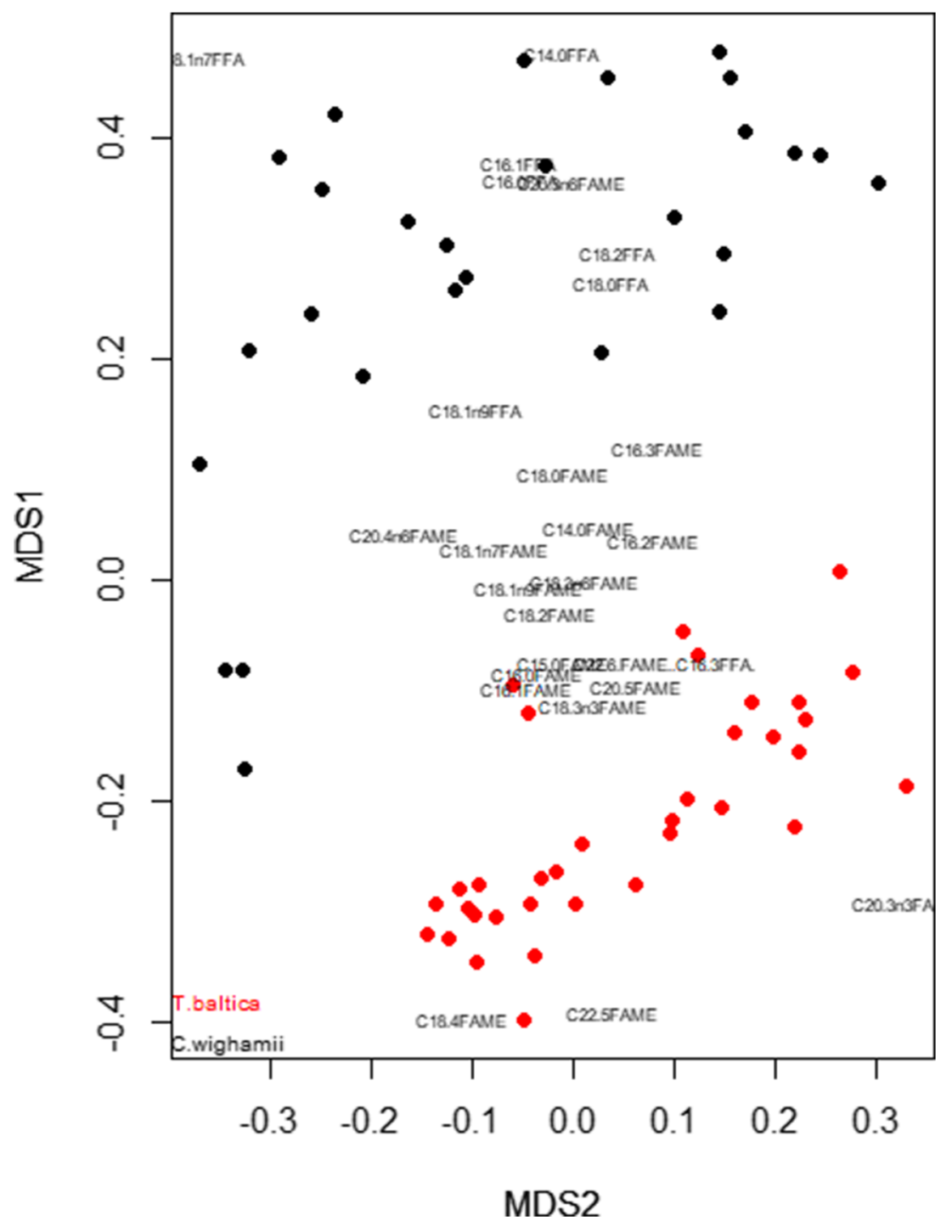

Considering commercial cultivation of these species, a central question is how well the EPA content of $T$. baltica compares to other alternative species. In the review by Jónasdóttir (2019), diatoms were pointed out as one of the best groups containing highly unsaturated FAs (HUFA) including EPA and DHA at $2.3 \pm 3.1 \%$ (SD) of total carbon content. According to our results, T. baltica contained up to $7.7 \pm$ $1.0 \%$ (SD) HUFA of the POC content under Si-limitation. The total biomass produced under these conditions was low, however, but the HUFA content was still $4.0 \pm 0.3 \%$ (SD) of POC on average in the N-limited biomass, which is still high compared with the numbers presented in Jónasdóttir (2019). The amount of EPA at $3 \%$ of AFDW was comparable to the best values obtained with P. tricornutum (Jiang and Gao 2004). Thalassiosira baltica had a higher share of EPA than comparable cold water diatoms (Artamonova et al. 2017; Steinrücken et al. 2017, 2018; Peltomaa et al. 2019), with the exception of Porosira glacialis that provides up to $10 \%$ 

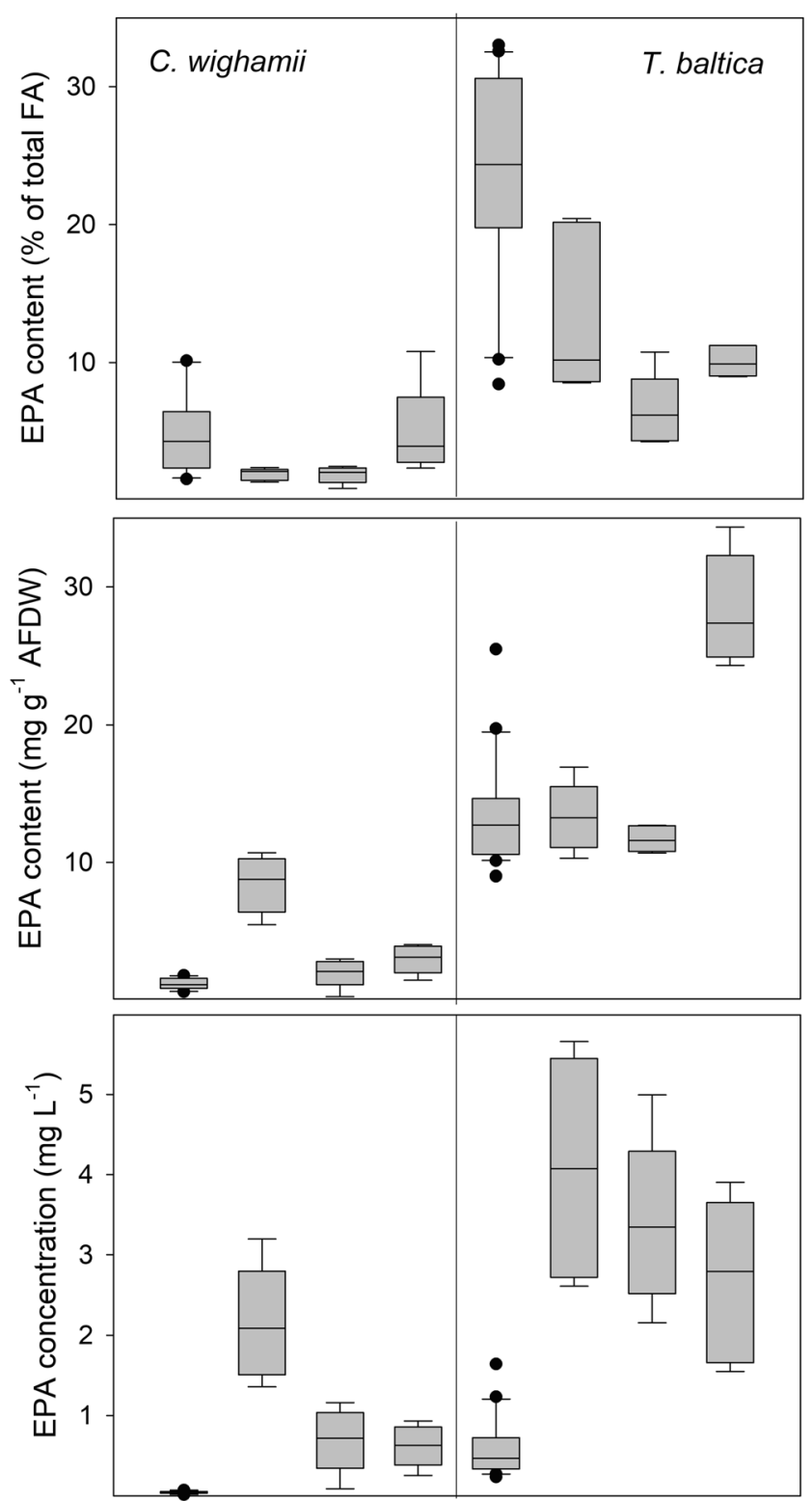

Exp N-lim P-lim Si-lim Exp N-lim P-lim Si-lim

Fig. 4 The eicosapentaenoic acid (EPA) content as a percentage of total fatty acids, as the content of ash-free dry weight (AFDW), and concentration harvested, for the two diatom species grown under exponential (Exp) and in stationary growth phase under nitrogen- $(\mathrm{N}$ lim), phosphorus- (P-lim), and silicate- (Si-lim) limitation. The box is the $25-75 \%$ percentile; the mid-line is the median; the whiskers indicate the $10-90 \%$ percentile and the dots outliers

EPA of total FAs (Svenning et al. 2019). Thalassiosira baltica could thus be a good candidate species for EPA production in a brackish, cold water cultivation system. However, Peltomaa et al. (2019) included a strain of T. baltica in their screening for FAs that had a much lower FA share overall, including EPA, and the suitability needs to be checked in individual strains.

In summary, the two cold water diatoms $C$. wighamii and T. baltica had very different FA profiles and the content of
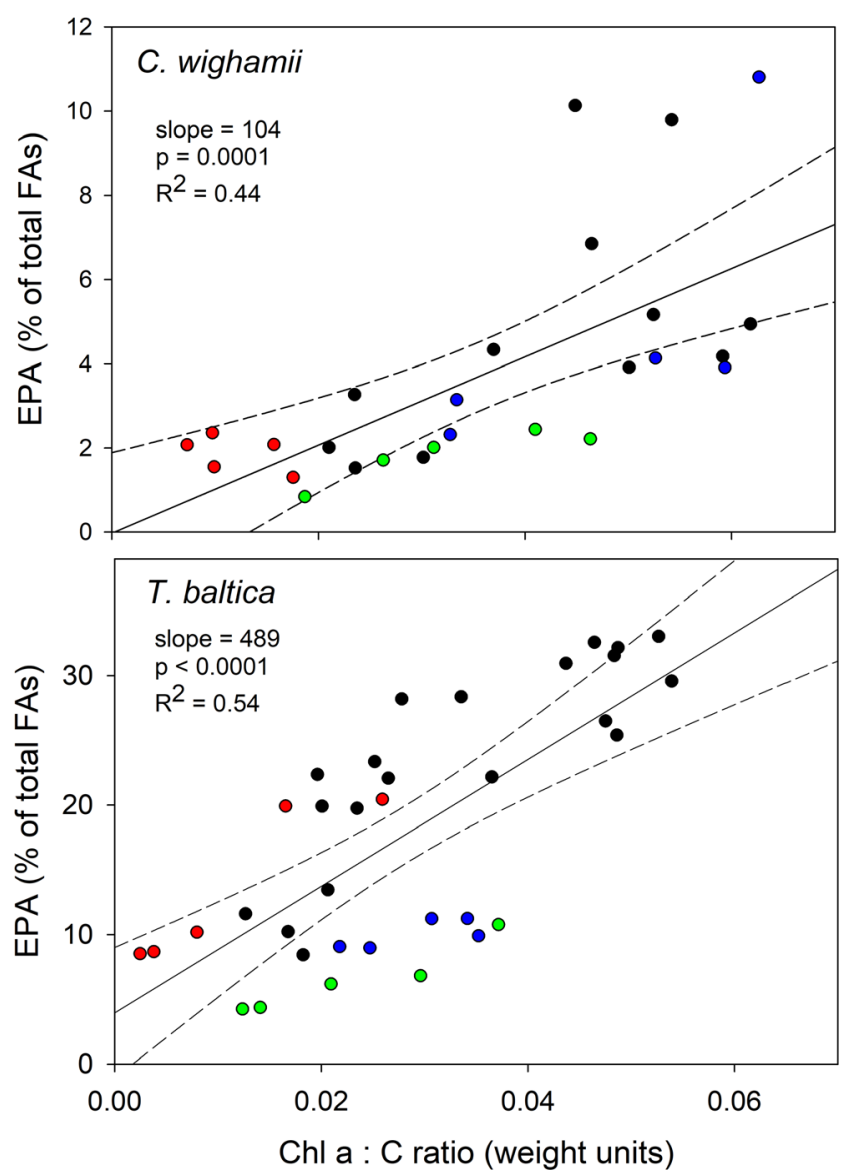

Fig. 5 The eicosapentaenoic acid (EPA) content as a percentage of total fatty acids (FAs) plotted against the $\mathrm{Chl} a$ :C ratio in Chaetoceros wighamii and Thalassiosira baltica during exponential (black), Nlimited (red), P-limited (green), and Si-limited (blue) growth. The black solid line is the linear regression and the dotted lines the $95 \%$ confidence interval

individual FAs was differentially affected by temperature, light, and nutrient limitation. Both species contained EPA, whereas the concentration of DHA was much lower in both species. Especially, T. baltica reached a high concentration of EPA up to levels comparable to the highest EPA producers found in the literature. The EPA content as a percentage of total FAs was positively correlated with the Chla:C ratio, suggesting that most of it is bound to membranes of the photosynthetic apparatus. However, higher biomass at high light compensated for a lower fraction of EPA and the total EPA content was not affected by the light level. Covering a wide range of environmental conditions, the strongest differentiation in FA profiles was observed between the species with the growth phase/nutrient limitation pattern as the second most important driver of the FA composition.

Supplementary Information The online version contains supplementary material available at https://doi.org/10.1007/s10811-021-02380-9. 
Acknowledgments We would like to thank the staff at the Marine Research Centre Laboratory for technical assistance and Riina Klais at EcoStat OÜ for help with the statistical analysis and data ordination plots. The study utilized SYKE-MRC marine research infrastructure as a part of the national FINMARI RI consortium.

Authors' contributions K.S., J.S., and T.T. organized and carried out the experiment. D.S. and H.R. carried out the measurements of fatty acids. K.S. wrote the manuscript with input from all other co-authors.

Funding Open Access funding provided by Finnish Environment Institute (SYKE). The project was funded by the Academy of Finland (grants 124733 and 124320).

Data Availability All data is included as supplementary material.

\section{Declarations}

Conflict of interest The authors declare that they have no conflict of interest.

Consent for publication All coauthors consent to the present version of the manuscript for submission for publication

Open Access This article is licensed under a Creative Commons Attribution 4.0 International License, which permits use, sharing, adaptation, distribution and reproduction in any medium or format, as long as you give appropriate credit to the original author(s) and the source, provide a link to the Creative Commons licence, and indicate if changes were made. The images or other third party material in this article are included in the article's Creative Commons licence, unless indicated otherwise in a credit line to the material. If material is not included in the article's Creative Commons licence and your intended use is not permitted by statutory regulation or exceeds the permitted use, you will need to obtain permission directly from the copyright holder. To view a copy of this licence, visit http://creativecommons.org/licenses/by/4.0/.

\section{References}

Artamonova E, Svenning J, Vasskog T, Hansen E, Eilertsen H (2017) Analysis of phospholipids and neutral lipids in three common northern cold water diatoms: Coscinodiscus concinnus, Porosira glacialis, and Chaetoceros socialis, by ultra-high performance liquid chromatography-mass spectrometry. J Appl Phycol 29:12411249

Brown MR, Dunstan GA, Norwood SJ, Miller KA (1996) Effects of harvest stage and light on the biochemical composition of the diatom Thalassiosira pseudonana. J Phycol 32:64-73

Camarena-Gómez MT, Ruiz-Gonzalez C, Piiparinen J, Lipsewers T, Sobrino C, Logares R, Spilling K (2021) Bacterioplankton dynamics driven by interannual and spatial variation in diatom and dinoflagellate spring bloom communities in the Baltic Sea. Limnol Oceanogr 66:255-271

Douce R, Joyard J (1996) Biosynthesis of thylakoid membrane lipids. In: Ort DR, Yocum CF, Heichel IF (eds) Oxygenic photosynthesis: the light reactions. Springer, Dordrecht, pp 69-101

Falkowski PG, Raven JA (1997) Aquatic photosynthesis. Blackwell Science, Oxford
Guillard RRL (1975) Culture of phytoplankton for feeding marine invertebrates. In: Smith WL, Chanley MH (eds) Culture of marine invertebrate animals. Plenum Press, New York, pp 26-60

Guschina I, Harwood J (2009) Algal lipids and effect of the environment on their biochemistry. In: Arts M, Brett M, Kainz M (eds) Lipids in aquatic ecosystems. Springer, New York, pp 1-24

GVR (2020) Omega 3 supplements market size, share \& trends analysis report by source (fish oil, krill oil), by form (soft gels, capsules), by functionality, by end-user, by distribution channel, by region, and segment forecasts, 2020-2027. Grand View Research report: GVR$1-68038-818-3$

Jespersen AM, Christoffersen K (1987) Measurements of chlorophyll- $a$ from phytoplankton using ethanol as extraction solvent. Arch Hydrobiol 109:445-454

Jiang H, Gao K (2004) Effects of lowering temperature during culture on the production of polyunsaturated fatty acids in the marine diatom Phaeodactylum tricornutum (Bacillariophyceae). J Phycol 40:651654

Jónasdóttir SH (2019) Fatty acid profiles and production in marine phytoplankton. Mar Drugs 17:151

Keitt TH (2008) Coherent ecological dynamics induced by large-scale disturbance. Nature 454:331-334

Khozin-Goldberg I (2016) Lipid metabolism in microalgae. In: Borowitzka MA, Beardall J, Raven JA (eds) The physiology of microalgae. Springer, Cham pp, pp 413-484

Koistinen J, Sjöblom M, Spilling K (2017) Determining inorganic and organic carbon. In: Spilling K (ed) Biofuels from algae. Methods in Molecular Biology, vol 1980. Humana, New York, pp 63-70

Lemon J (2006) Plotrix: a package in the red light district of R. R-News 6:8-12

Liang Y, Koester JA, Liefer JD, Irwin AJ, Finkel ZV (2019) Molecular mechanisms of temperature acclimation and adaptation in marine diatoms. ISME J 13:2415-2425

Nalewajko C (1966) Dry weight, ash, and volume data for some freshwater planktonic algae. J Fish Res Board Can 23:1285-1288

Peltomaa E, Hällfors H, Taipale SJ (2019) Comparison of diatoms and dinoflagellates from different habitats as sources of PUFAs. Mar Drugs 17:233

Schwenk D, Seppälä J, Spilling K, Virkki A, Tamminen T, OksmanCaldentey K-M, Rischer H (2013) Lipid content in 19 brackish and marine microalgae: influence of growth phase, salinity and temperature. Aquat Ecol 47:415-424

Spilling K (2007) On the ecology of cold-water phytoplankton in the Baltic Sea. PhD thesis, Univ Helsinki. W \& A de Nottbeck Foundation Scientific Reports 31:1-59

Spilling K, Ylöstalo P, Simis S, Seppälä J (2015) Interaction effects of light, temperature and nutrient limitations $(\mathrm{N}, \mathrm{P}$ and $\mathrm{Si})$ on growth, stoichiometry and photosynthetic parameters of the cold-water diatom Chaetoceros wighamii. PloS One 10:e126308

Steinrücken P, Erga SR, Mjøs SA, Kleivdal H, Prestegard SK (2017) Bioprospecting North Atlantic microalgae with fast growth and high polyunsaturated fatty acid (PUFA) content for microalgae-based technologies. Algal Res 26:392-401

Steinrücken P, Mjøs SA, Prestegard SK, Erga SR (2018) Enhancing EPA content in an arctic diatom: a factorial design study to evaluate interactive effects of growth factors. Front Plant Sci 9:491

Svenning JB, Dalheim L, Eilertsen HC, Vasskog T (2019) Temperature dependent growth rate, lipid content and fatty acid composition of the marine cold-water diatom Porosira glacialis. Algal Res 37:1116

Swanson D, Block R, Mousa SA (2012) Omega-3 fatty acids EPA and DHA: health benefits throughout life. Adv Nut 3:1-7 
Thompson PA, Guo M, Harrison PJ, Whyte JN (1992) Effects of variation in temperature. II. On the fatty acid composition of eight species of marine phytoplankton. J Phycol 28:488-497

Williams PJLB, Laurens LML (2010) Microalgae as biodiesel \& biomass feedstocks: review \& analysis of the biochemistry, energetics \& economics. Energy Env Sci 3:554-590

Wood S (2016) mgcv: GAMs with GCV/AIC/REML smoothness estimation and GAMMs by PQL. R package version 1:8-16
Xu J, Chen D, Yan X, Chen J, Zhou C (2010) Global characterization of the photosynthetic glycerolipids from a marine diatom Stephanodiscus sp. by ultra performance liquid chromatography coupled with electrospray ionization-quadrupole-time of flight mass spectrometry. Anal Chim Acta 663:60-68

Publisher's note Springer Nature remains neutral with regard to jurisdictional claims in published maps and institutional affiliations. 